\title{
Du village à la ville : les trajets professionnels des marins de la Seudre (1770-1793)
}

\section{Thierry Sauzeau}

\section{(2) OpenEdition}

\section{Édition électronique}

URL : http://journals.openedition.org/abpo/541

DOI : 10.4000/abpo.541

ISBN : 978-2-7535-1504-8

ISSN : 2108-6443

\section{Éditeur}

Presses universitaires de Rennes

Édition imprimée

Date de publication : 30 décembre 2006

Pagination : 83-96

ISBN : 978-2-7535-0405-9

ISSN : 0399-0826

\section{Référence électronique}

Thierry Sauzeau, «Du village à la ville : les trajets professionnels des marins de la Seudre

(1770-1793) », Annales de Bretagne et des Pays de l'Ouest [En ligne], 113-4 | 2006, mis en ligne le 30 décembre 2008, consulté le 21 avril 2019. URL : http://journals.openedition.org/abpo/541 ; DOI : 10.4000/abpo.541 


\title{
Du village à la ville : les trajets professionnels des marins de la Seudre (1770-1793)
}

\author{
Thierry SAUZEAU \\ Maître de conférences en histoire moderne \\ GERHICO - Université de Poitiers
}

La participation française à la première forme d'économie monde, qui court du XVI ${ }^{\mathrm{e}}$ au milieu du XIX ${ }^{\mathrm{e}}$ siècle, a mené toute une frange des hommes du littoral sur les routes de la morue, du sucre et des esclaves. Objet de grandes thèses d'histoire économique, la géographie des destinations de la marine à voile est connue. Cette thématique a bénéficié de la vague de l'histoire quantitative, malheureusement retombée avant d'avoir atteint un $\mathrm{XIX}^{\mathrm{e}}$ siècle encore à inventer ${ }^{1}$. Depuis une quinzaine d'années, c'est dans le champ de l'histoire sociale que se sont investies les énergies : l'histoire des gens de mer d'Ancien Régime a ainsi été profondément renouvelée. Les migrations induites par l'activité maritime moderne peuvent s'appréhender à trois échelles. Définitives, elles concernent engagés et émigrants mais aussi les marins déserteurs du commerce. Temporaires, elles épousent la géographie du commerce qui amène les matelots à visiter des destinations exotiques avant de revenir chez eux. Ces deux réalités du métier maritime sont assez aisées à mesurer, tant le destin des individus s'y confond exactement avec celui des navires. Tel n'est pas le cas d'autres voyages, impliquant uniquement les marins non citadins des grands ports. Ce troisième niveau de déplacement, temporaire et lié au travail, concerne les gens de mer ruraux, qui gagnaient régulièrement la ville portuaire voisine pour y trouver à s'embarquer. Ils imitaient un peu les Auvergnats, quittant leurs vallées pour vendre leur savoir-faire à Paris ${ }^{2}$. En Saintonge maritime, entre La Rochelle et Bordeaux, le quartier de Marennes a ainsi vu ses marins, au nombre de 6 à 900 dans la seconde moitié du XVIII ${ }^{\mathrm{e}}$ siècle, se partager entre

1. C'est ce que rappellent non sans raison Sylviane Llinares et Gérard Le Bouëdec dans "Les arsenaux face aux enjeux géostratégiques atlantiques (XVII -XIX ${ }^{\mathrm{e}}$ siècles)", dans Enjeux maritimes des conflits européens (XVI'-XIX siècles), Documents \& Enquêtes $\mathrm{n}^{\circ} 28$, CRHMA, Nantes, Ouest-Éditions, 2002, p. 149-167.

2. POITRINEAU, Abel, Remues d'hommes, les migrations campagnardes en France, XVII XIXe siècles, Paris, Aubier, 1983. 
les quais des grands ports voisins. L'étude de ces trajets, déplacements " préparatoires " au voyage de mer, peut-être entreprise grâce aux archives du système des Classes. Elle met en lumière un aspect méconnu du quotidien. C'est par la performance d'un modeste échelon de la monarchie administrative, le bureau des " classes ", qu'il importe d'entamer cette présentation. Les archives qu'on y a produites permettent en effet de dresser la géographie des déplacements vers les villes. L'apport de la source ne s'arrête pas là puisqu'elle permet aussi d'envisager la chronologie des voyages et ses facteurs.

\section{Un quotidien révélé : les registres matricules des " classes "}

Sur le littoral comme dans le reste du royaume, le règne de Louis XIV a laissé son empreinte. C'est alors qu'on a confié à des hommes neufs la charge de représenter le Roi. Dans les provinces, l'intendant de police, justice et finances, au chef-lieu d'une généralité, était à la tête d'un réseau de subdélégués dans chaque élection. La grande ordonnance de la marine devait appliquer au littoral ces mêmes principes. Reléguant le monde des officiers d'amirauté dans un rôle d'hommes de loi des côtes, ce texte organisait en fait le triomphe d'une hiérarchie de commissaires. Sous l'autorité du ministère de la marine et des colonies, on procédait à la division du littoral en départements, à la tête desquels l'intendant de marine était relayé dans chaque quartier par un commissaire. Le succès de l'enrôlement des gens de mer saintongeais, dès septembre 1668, provoquait la généralisation à l'ensemble du pays l'année suivante ${ }^{3}$. L'objectif du système des classes était d'organiser un service militaire à tour de rôle pour tous ceux qui faisaient profession de naviguer ou s'adonnaient à des industries maritimes (charpentiers, voiliers, cordiers, poulieurs). En théorie, répartis en trois classes sur le littoral picto-charentais, les gens de mer devaient être appelés au service sur les navires de guerre à Rochefort une année sur trois. La guerre devait endommager gravement l'institution, en piteux état à la mort du Roi-Soleil (1715). Il fallut attendre les années 1724-1727, pour que le ministre Maurepas remette en vigueur la discipline des classes. À Cherbourg, la plus ancienne série de registres conservée date des années 1726-1735. Ce registre de matelots compte 77 inscrits n'ayant jamais été classés comme mousses alors qu'un demi-siècle plus tard, la série 17751785 n'en compte plus que deux ${ }^{4}$. À partir du milieu du XVIII ${ }^{\mathrm{e}}$ siècle, l'habitude de tenir des registres matricules s'est donc pérennisée, notre vision des carrières individuelle s'en trouve clarifiée.

À Marennes, le commissaire aux classes avait pour mission de gérer la population d'inscrits d'un quartier centré sur l'estuaire de la Seudre. Son

3. ZYSBERG, André, « La soumission du rivage aux volontés de l'État Royal ", dans État, Marine et Société, Hommage à Jean Meyer, Paris, PU Sorbone, 1995, p. 440.

4. Selon Carine Blanc, Les Gens de mer de Fermanville au XVIII siècle, Mémoire de mâ̂trise, Université de Paris X, 1989, p. 43 (sous série 4P6). 
travail avait ceci de particulier qu'il gérait une population fixe et mobile à la fois. L'éloignement entre lieux de travail (Bordeaux, La Rochelle) et lieux de résidence (pays de la Seudre) impliquait le recours à une abondante paperasse. Soucieuse de lutter contre le vagabondage d'une population gyrovague par excellence, la grande ordonnance de la marine avait instauré l'obligation du "bulletin ", appelé " permis " à la fin du XVIII siècle. Ce document délivré à Marennes, était un laissez-passer permettant aux inscrits du quartier de le quitter pour se rendre en ville. La loi punissait l'employeur d'un marin sans permis. Afin de garder une trace de ce travail quotidien, le bureau disposait d'une main courante nommée " journalier " où l'on notait les permis délivrés mais aussi les levées au service. Périodiquement, les bureaux d'armement (La Rochelle, Bordeaux, Rochefort - Marine) envoyaient des listes de marins enrôlés sur leurs quais mais inscrits à Marennes. Au terme d'une campagne ou d'un voyage, l'envoi de listes de désarmement donnait les noms, prénoms et matricules des marins renvoyés, munis d'un congé, dans leurs foyers. À l'issue de son voyage, chaque marin devait venir " accuzer son retour " au bureau. Toutes les informations étaient temporairement consignées dans le journalier. C'est finalement ce flot continu de papiers et courriers que le bureau de Marennes traitait afin de former les registres matricules. Si une pièce manquait, on interrogeait le syndic des gens de mer, représentant traditionnel des marins auprès des autorités. Au total, 22 villages possédaient leur syndic des gens de mer autour de la Seudre.

Envoyée vierge dans chaque quartier, chaque série de registres était ouverte pour une période définie ${ }^{5}$. Des volumes d'apprentis (mousses, novices) contenaient un état nominatif des inscrits rangés par paroisse et établi dans l'ordre chronologique d'inscription. Après avoir rempli un cartouche préimprimé mentionnant l'état civil, le classement au service et les éventuels antécédents de l'inscrit, l'officier remplissait au gré des années, les activités civiles (pêche, cabotage, long cours) ou militaires (service, course). À la faveur d'une revue d'effectifs, d'une promotion au service ou d'une nouvelle série de registres, on rayait le cartouche sur l'ancien registre avant d'en établir un sur le nouveau. Il existait aussi des registres de matelots et officiers mariniers, de capitaines, de maîtres, de pilotes ou d'ouvriers. Sur les feuilles, dont la présentation a évolué entre 1760 et 1850, chaque inscrit était repéré par un numéro de page (le folio) et un numéro de cartouche (le numéro). Ces deux références ainsi que celle de sa paroisse et de son quartier d'inscription étaient les identifiants, reportés

5. Carine Blanc dans : Les Gens de mer..., op. cit., remarque au SHM de Cherbourg cinq séries de registres à compter de la reprise en main par Maurepas (1726-1739; 1740-1749; 1750-1764; 1764-1775 et 1776-1787), tandis que chez PAROT-ABELLARD, Franck, Les Gens de mer classés sur les registres de la Marine royale : dénombrement et cartographie entre Loire et frontière espagnole, Mémoire de maîtrise, Université de Poitiers, 1997, p. 18-20, la présentation des sources des quartiers de l'intendance de Rochefort met en lumière l'existence des séries 1764-1775, 1776-1787, 1788-1796, 1796-an XII, an XIII-1815, 1815-1825 (ou 1827) et $1828-1845$. 
sur le permis, les rôles d'équipage (armement/désarmement) et le congé de retour.

Marqués par une antériorité des pratiques (1668), les registres saintongeais sont bien tenus et leur collection sans lacune insurmontable permet la reconstitution de carrière des inscrits, en suivant chacun d'un registre à un autre. Tenue de manière idéale, cette source permet d'étudier les rythmes de travail, en donnant quatre repères précis en matière chronologique : le permis, l'embarquement, le débarquement, le retour au quartier. On peut donc y rechercher les logiques, individuelles ou collectives, de tels trajets professionnels. Par leur finesse, les sources permettent de lever le voile sur la chronologie de ces déplacements, que les gens de mer de Saintonge réalisaient vers Rochefort ${ }^{6}(30 \mathrm{~km})$, La Rochelle $(60 \mathrm{~km})$ ou Bordeaux (150 km).

\section{Où partir? La Seudre, carrefour de bassins d'emplois}

Riche de son sel, la Seudre a dû intégrer très tôt les grands circuits d'échange médiévaux, dont la Saintonge viticole et la région voisine de Bordeaux étaient des pôles importants. Rouliers du golfe de Gascogne, de l'Espagne à la Manche, les marins saintongeais se sont investis très tôt dans l'aventure de la grande pêche, sur leurs propres navires comme au service des Rochelais ${ }^{7}$. Sous le règne de Louis XIV, à une époque où la puissance bordelaise n'était qu'en gestation, les matelots du quartier de Marennes remontaient aussi la Gironde jusqu'aux quais de la Garonne pour s'embarquer sur les long-courriers ${ }^{8}$. À la fin du XVIII ${ }^{\mathrm{e}}$ siècle, la Seudre confortait donc un rôle de "pays naisseur " de matelots pour le long cours atlantique. Aussi convient-il à présent de mettre en perspective la participation des marins du quartier aux entreprises commerciales concurrentes de Bordeaux et La Rochelle - Rochefort. Les marins saintongeais prenaient régulièrement le chemin des grands ports du Centre-Ouest mais qu'en étaitil des zones d'attraction concurrentes de l'Aunis et de la Gironde?

Bordeaux puisait largement dans les forces vives du quartier ${ }^{9}$. En 1772, les registres signalaient 245 inscrits du quartier enrôlés à Bordeaux où l'ensemble de la flotte de long cours nécessitait 1954 marins. En Aunis, l'effort

6. Sur l'activité du port de commerce de Rochefort, voir notre article « Rochefort et l'Amérique (XVII $-\mathrm{xIX}^{\mathrm{e}}$ siècle) ", dans AugERON, Mickaël et HuERTA, Mona (dir.), La Rochelle et Rochefort, les Amériques en partage. Ressources documentaires, Karthala, à paraître printemps 2005

7. SEGuIN, Marc, "Les débuts de la pêche saintongeaise à Terre Neuve (1546-1570) ", dans Les sociétés littorales du Centre Ouest atlantique, Rochefort 1995, Poitiers, SAO, 1998.

8. HuETz de LEMPS, Christian, Géographie du commerce de Bordeaux à la fin du règne de Louis XIV, Paris/La Haye, Mouton, 1975.

9. Butel, Paul, Les Négociants bordelais au XVII' siècle. L'Europe et les Îles, Paris, Aubier, 1974 , p. 221, indique qu'en 1772, les équipages bordelais étaient composés de $25 \%$ de marins de la cité qui recrutait encore $22 \%$ de Saintongeais (Quartiers de Marennes, Royan et Oléron). 
humain s'élevait à 37 en cette même année alors que La Rochelle et Rochefort avaient besoin de 315 marins. Le quartier fournissait donc un peu plus de $10 \%$ des marins girondins et aunisiens. La question des modalités de ce " partage " inégal de la main d'œuvre entre l'Aunis et Bordeaux doit être envisagée. La Rochelle était en fait le vivier principal de ses propres équipages (60\%) et, comme dans le cas de Bordeaux ${ }^{10}$, plus de la moitié des marins de la cité étaient cadres. Logiquement, les Rétais fournissaient le plus important contingent horsain (20\%). Les fameuses " aires concentriques de bassins migratoires de plus en plus larges ${ }^{11}$ " étaient ensuite à l'œuvre. Vers le nord, l'attraction du port de Nantes se faisait sentir jusqu'au bas Poitou ${ }^{12}$. C'est pourquoi les équipages rochelais ne recrutaient guère au nord d'une ligne Marans-Les Sables-d'Olonne. Vers le sud, la situation était plus confuse puisque Bordeaux étendait son empire sur la population active de la Saintonge " girondine " (de Mortagne à SaintPalais) comme sur l'estuaire de la Seudre. Employeurs rochelais et bordelais entraient donc en concurrence dans le quartier de Marennes où la Seudre jouait le rôle de ligne de partage des bassins d'emplois. Ainsi, Marennes et Bourcefranc-le-Chapus, sur la rive nord, étaient le troisième foyer de main d'œuvre en Aunis. Au sud de la Seudre, seuls quelques Trembladais et les gens de Chaillevette les imitaient. Les Isles de Marennes, prolongées par Oléron, étaient donc humainement orientées vers l'Aunis tandis que les Isles d'Arvert étaient, à l'instar de Royan et sa région, un avant-port humain de Bordeaux. Année moyenne avec 354 marins du quartier enrôlés à Bordeaux, 1785 peut fournir une bonne base de cartographie de l'origine, village par village, des marins embarqués sur des navires armés au long cours en Gironde. Les équipages bordelais puisaient dans la population active du quartier, étendant le recrutement jusque dans des villages paysans de la terre ferme comme Saint-Sulpice, Médis et Breuillet dans l'isthme royannais, ou encore Les Mathes et Étaules en Arvert. Ces foyers ruraux de migrants temporaires étaient cependant modestes et composés presque uniquement de matelots, fils de paysans pour la plupart. De même, le niveau de qualification des Marennais partant vers la Gironde était plus faible que celui des migrants vers l'Aunis. C'était tout le contraire des marins venus des villages de l'estran salicole des Isles d'Arvert. Un peu plus du tiers des marins issus de La Tremblade-Chaillevette étaient officiers mariniers du long cours bordelais. Autour de ces deux pôles, apparaissaient de nombreux villages. Arvert et ses villages (Avalon, Fouillou) fournissaient $20 \%$ des migrants du quartier vers Bordeaux tandis que, de Mornac à Saujon-Le Gua, les villages du fond de l'estuaire, participaient à hauteur de $18 \%$.

10. HuETz de LEMPS, Christian, Géographie du commerce..., op. cit., carte $\mathrm{n}^{\circ} 34$.

11. CaBantous, Alain, Les Citoyens du large. Les identités maritimes en France (XVII - XIX ${ }^{e}$ siècles), Paris, Aubier, coll. " Historique ", 1995, p. 168.

12. Voir à ce sujet l'article de BOUYER, Murielle, "L'attraction progressive de Nantes sur les petits ports des littoraux bretons et poitevins (1694-1715) ", dans Des villes, des ports, la mer et les hommes, Nantes, colloque 1999, CTHS, 2001. 
La Seudre était bien la ligne de partage entre les bassins d'emploi girondin et aunisien. Les cadres et les matelots de Marennes et Bourcefranc-leChapus partaient vers La Rochelle. Au sud de la Seudre, La Tremblade ou Chaillevette et ses nombreux villages disposaient d'une tradition humaine comparable : ces villages envoyaient d'importants contingents d'officiers mariniers et majors vers Bordeaux. Les Isles d'Arvert figuraient une véritable banlieue humaine de Bordeaux. Deux dynamiques humaines de directions opposées, mais finalement très semblables dans leur forme, amenaient les habitants du littoral saintongeais à se déplacer vers les quais du long cours en Aunis ou à Bordeaux.

\section{Partir à tout prix? Départs choisis, départs subis}

Au service d'une économie mondialisée, les gens de mer ruraux de Saintonge voyaient leur recherche dirigée par l'offre d'emploi. La force de l'appel n'excluait pourtant pas une certaine forme de régulation interne, au niveau familial et social. Apprentis, matelots ou cadres de la navigation de long cours, tous ne réagissaient pas à l'identique, en fonction de leurs origines et des mutations du métier.

Grâce aux travaux de Paul Butel ${ }^{13}$, on a une idée précise de l'offre d'emplois sur les rives de la Garonne à compter de l'année 1760 et, pour La Rochelle-Rochefort, on peut se baser sur les collections de rôles d'armement, complétés par le registre des soumissions de l'Amirauté de La Rochelle ${ }^{14}$. Ce n'est finalement qu'à compter de 1770 que l'on peut s'appuyer sur une image de classes complètes ${ }^{15}$. Rappelons que l'appel de main d'œuvre maritime à Bordeaux était six à sept fois plus intense qu'en Aunis, toute proche. Les quais de Bordeaux voyaient s'enrôler d'importants contingents de marins de la Seudre qui, compte tenu de la durée d'un voyage, représentaient chaque année une fraction allant de $30 \%$ à $80 \%$ de la population active du quartier. À Bordeaux, la participation humaine de la Saintonge maritime suivit la croissance du trafic jusqu'à la guerre d'Indépendance Américaine. Passé la guerre, l'effort humain du Quartier ne suivait plus le rythme imposé par les armements girondins. À partir de 1784, la Seudre n'envoyait plus qu'un peu plus de 300 marins chaque année, ce qui représentait 35 à $55 \%$ de sa population active. En fait, si les matelots partaient toujours en masse vers le " port de la lune ", ils n'étaient plus suivis de ces amples moissons d'apprentis qui avaient fait la renommée de " pays naisseur " de la Saintonge maritime.

13. BuTEL, Paul, op. cit., graphique p. 391

14. Arch. dép. de Charente-maritime, fonds de l'Amirauté de La Rochelle, B.259. Présenté en annexes par DEvEAU, Jean-Michel, Le commerce rochelais face à la Révolution. Correspondance de Jean-Baptiste Nairac, La Rochelle, Rumeur des âges, 1989.

15. Des apprentis aux retraités, les hors de service, en passant par les matelots et les cadres. 
Finalement, à la fin du XVIII ${ }^{\mathrm{e}}$ siècle, en Seudre, le modèle de reproduction sociale connaissait une sérieuse panne qui invite à analyser l'apprentissage. Un apprenti (mousse ou novice) sur deux sortait habituellement d'une famille de paysans, de sauniers ou d'artisans. La carrière maritime était cependant un risque différemment appréhendé suivant les milieux. On l'abordait avec confiance dans les familles maritimes, où $64 \%$ des fils de capitaines et $68 \%$ des garçons dans les milieux populaires devenaient marins. On manifestait davantage de défiance chez les terriens : la bourgeoisie ne destinait que $45 \%$ de ses fils à la navigation et les milieux populaires $48 \%$. L'examen du rang de naissance ${ }^{16}$ apporte son éclairage complémentaire. Dans les familles maritimes, on envoyait les aînés naviguer le plus tôt possible, comme mousses. La réputation au sein du métier était un capital qu'il ne fallait pas laisser échapper. En revanche, chez les acteurs de l'économie rurale, c'étaient les cadets qui intégraient, le plus tard possible, la navigation : on les retrouvait en masse parmi les novices. Ici, on demandait à la mer d'absorber les bras dont on ne savait que faire dans les campagnes ou sur les marais. C'est la fragilité de cet équilibre entre confiance et défiance que la mutation économique de la décennie 1780 a brisée en Seudre.

Dans les années 1770, moins de $5 \%$ des embarquements offerts à Bordeaux concernaient la traite négrière ou l'Inde. On passait à $13 \%$ des postes au cours de la décennie suivante, Bordeaux s'affirmant comme port négrier ${ }^{17}$ à l'instar de Nantes ou La Rochelle. Ce type de contrat était synonyme de recul social puisque les négriers étaient rarement des navires neufs, que les capitaines n'y faisaient pas carrière et que la moitié d'entre eux étaient des néophytes ${ }^{18}$. À Bordeaux, les gens de mer saintongeais se retrouvaient dans la même situation que les Bretons enrôlés à Nantes ${ }^{19}$, contraints d'accepter de tels postes, boudés par la main d'œuvre locale. Cette inflexion économique pouvait être de nature à éloigner les jeunes de la Seudre du métier d'autant que l'étroit marché du travail de l'Aunis ne donnait pas de réelle alternative. C'est dès après la guerre de Sept Ans et la perte du Canada que la cité aunisienne se tourna d'abord timidement puis plus franchement vers le commerce triangulaire. Le commerce rochelais des années 1780 était en fait dominé par l'armement en traite qui passa de 25 à $30 \%$ des expéditions avant guerre à une part dominante, entre 50 et $70 \%$ après. Cette évolution ne pouvait manquer de faire glisser le centre de gravité de la profession entière vers le pont des navires négriers. Pour les matelots de la Seudre, cela signifiait la nécessité d'investir cet espace professionnel nouveau car les matelots de La Rochelle et de l'île de Ré, à

16. Voir notre article, "L'apprentissage des gens de mer à la fin du XVIII siècle ", dans Techniques \& Cultures, $\mathrm{n}^{\circ}$ spécial "Formation et apprentissage ", Paris, CNRS, à paraître.

17. L'ouvrage de SAUGERA, Éric, Bordeaux, Port négrier, XVIe-XIXe siècles, Paris, Karthala, 1995, a insisté sur ce passé méconnu de la cité commerçante.

18. Ibidem, p. 225 et 241-42.

19. Suivant BOUYER, Murielle, "L'attraction progressive de Nantes... ", art. cit., p. 51, 50 \% des matelots de la traite étaient bretons. 
la manière des Nantais, se réservaient les voyages en droiture. Dans les enrôlements des gens de mer de la Seudre, les embarquements sur des négriers devenaient deux fois plus fréquents. À côté de la Guinée, la Côte de l'Or ou du Sénégal, de nouvelles destinations, la côte d'Angolle ainsi que le Mozambique et la côte d'Afrique, au-delà du cap de Bonne Espérance apparaissaient après la guerre d'Indépendance Américaine. L'économie servile se développait aux Îles de France et de Bourbon et la côte d'Afrique orientale payait son tribut d'esclaves aux plantations. Les gens de mer de la Seudre se trouvaient donc, malgré eux, engagés dans un front pionnier à risque de l'économie monde.

La crise de recrutement enregistrée dans les années 1780 était donc sans doute le résultat de facteurs qui se relayaient et cumulaient parfois leurs effets. C'était peut-être le traumatisme de la guerre maritime, vivement ressenti en raison de l'absence prolongée des pères, frères ou époux, au service du Roi. C'était plus sûrement la croissance de l'armement négrier dans les ports du Centre-Ouest, qui mettait les marins de la Seudre dans l'obligation d'accepter ce type de contrat. Si les matelots hésitaient à se reconvertir, les plus jeunes ne prenaient plus la route de la ville lorsque les risques du métier devenaient trop grands.

\section{S’embarquer en ville : le résultat d'un trajet réussi}

Entre 1770 et 1793, les marins du quartier répondaient à l'appel des armateurs bordelais (84,5\% des embarquements), rochelais (10,2\%) ou rochefortais (4,2\%) : seuls $1,1 \%$ des trajets s'orientaient vers un autre port (Nantes, Marseille, Le Havre, Lorient). Prenant la route dans un environnement professionnel polarisé, l'individu devait encore tout mettre en œuvre pour conclure son trajet par l'enrôlement espéré.

Pour l'immense majorité des gens de mer, mousses, novices, matelots ou membres de la maistrance, la question des réflexes collectifs face au départ vaut d'être sondée. Dès la prise de permis, on pouvait repérer ceux qui étaient mus par le projet cohérent de partir en équipe autour d'un meneur, intermédiaire entre le vivier saintongeais et les employeurs au long cours. À bord, on partagerait le hamac et les quarts qu'il fallait prendre alternativement avec un autre marin. À l'époque, c'est le capitaine et son maître d'équipage qui amatelotent tout l'équipage avant d'embarquer, associant les hommes par paire : l'un sera de quart quand l'autre dormira ${ }^{20}$.

Entre 1770 et 1793, les registres matricules permettent d'identifier les équipes qui étaient constituées au minimum d'un matelot (deux marins). L'embarquement en solitaire au sein d'un équipage formé de marins étrangers à la Seudre était un cas minoritaire, limité à 27,3 \% des inscrits. Par ailleurs, l'amatelotage strict (deux marins de la Seudre à bord) ne concer-

20. Matelot serait un mot venu du hollandais matenoot, que l'on peut traduire par " compagnon qui partage la même couche ". Ces détails et bien d'autres sont extraits de MERRIEN, Jean, La Vie des marins au grand siècle, Rennes, Terre de Brume, 1995, p. 91-92. 


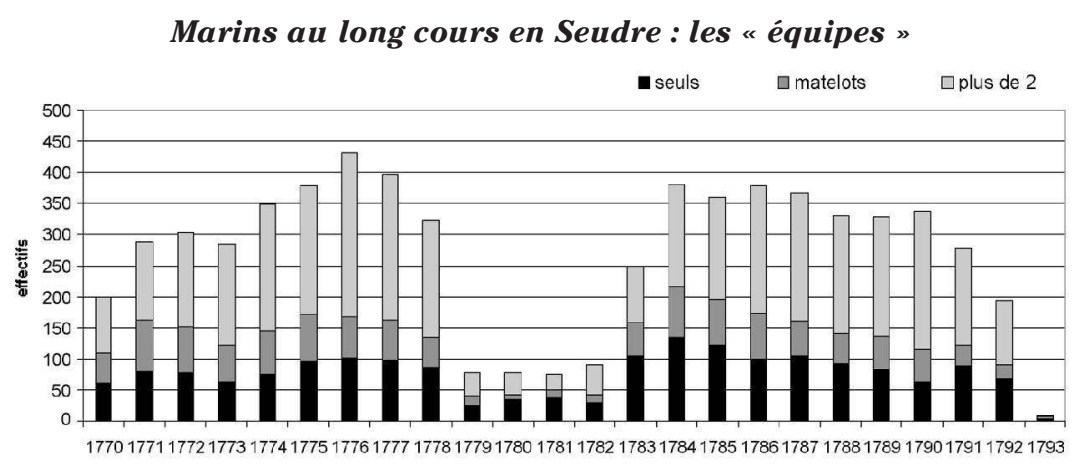

nait que $17,8 \%$ des individus, ce qui revient à dire que 54,9\% des marins du quartier de Marennes enrôlés au long cours étaient trois ou plus sur le même navire. Cette habitude de partir en groupe vers la ville était révélatrice de solidarités villageoises ou professionnelles. Avec ses 478 embarquements réalisés sur 170 navires différents, l'année 1776 permet de faire le point sur les profils sociologiques associés aux différents comportements. La composition du groupe de 82 marins venus isolément compléter un équipage faisait la part belle aux novices ( $46 \%$ ) et aux matelots sans spécialité (34\%). Il s'agissait par voie de conséquence d'une population marquée par des origines terriennes (56\%). Cumulant des handicaps sociaux et professionnels, ces hommes étaient sans doute maintenus à l'écart des conversations, s'intégraient moins bien et ne bénéficiaient pas des réseaux de solidarité à l'œuvre chez les matelots comme dans les équipes plus larges. Fils d'un boucher d'Arvert, Pierre Royer (Arvert, né en 1754, marin de 1775 à 1780) ${ }^{21}$ prenait ainsi son permis à Marennes le 6 février 1776 avant d'embarquer le 23 sur Le Grand Rivage, capitaine Dematerre, pour un voyage aux Îles. Sans le soutien d'autres marins de sa région, ce fils d'artisan illustrait assez bien les difficultés rencontrées par les terriens dans leur volonté d'intégration au métier de marin du long cours, fin XVIII ${ }^{\mathrm{e}}$ siècle. Cette faiblesse était à l'origine de l'absence d'embarquements à La Rochelle, car l'anonymat du gigantesque armement bordelais était sans doute préférable pour tenter sa chance.

La participation collective à une expédition de long cours, préparée à l'avance, intégrait moins de novices (40\%) et de matelots sans spécialité (30\%) car c'était le cadre privilégié de l'initiation des mousses (12\%). S'il n'était pas exclusif, loin s'en faut, le recrutement était dominé et organisé par les fils de marins (53\%). L'embarquement en matelot concernait 64 marins du quartier en 1776 (27\% de l'effectif) et les associations qui étaient formées dans de telles occasions étaient assez significatives. Cette forme minimale de projet collectif était favorable à l'intégration des apprentis.

21. Service historique de la Rochefort (SHM), Registre des novices de Marennes (17641787), 10P3.4 $\mathrm{f}^{\circ} 405 \mathrm{n}^{\circ} 18$ et suiv. 
Mousses et novices étaient souvent parrainés par un matelot. Matelot bourcefrançais et fils de marin, Michel Bertin (Bourcefranc, 1752-1765 à 1788) ${ }^{22}$ prenait un permis pour Bordeaux le $1^{\mathrm{er}}$ août 1776 en compagnie de Pierre Grimard (Bourcefranc, 1749-1776 à 1780) ${ }^{23}$, fils de jardinier de deux ans son aîné, mais fraîchement inscrit comme novice. Sous la conduite du plus expérimenté, les deux hommes étaient enrôlés par le capitaine Grandin, sur La Bien Aimée, à destination des Îles. On saisit bien ici le mécanisme qui pouvait, année après année, permettre le renouvellement des classes de la marine, par synergie entre les individus de culture maritime et les autres. Projeté dès avant l'embarquement, l'amatelotage était aussi pratiqué par le personnel qualifié. Officiers mariniers ou d'état major emmenaient avec eux des apprentis ou des matelots de confiance. Alors qu'il n'était encore que lieutenant, le futur capitaine Pierre Fayaud (La Tremblade, $1748 ; 1763$ à 1791) ${ }^{24}$ prenait un permis pour Bordeaux le 20 mars 1776 en compagnie de Jean-Pierre Taupier (La Tremblade, 1760; 1776 à 1782) ${ }^{25}$, bientôt pilotin sur La Fortune du capitaine Rocher. Au-delà d'un simple amatelotage projeté dès le départ du quartier, la majorité des marins qui partaient s'embarquer à Bordeaux ou La Rochelle le faisaient au sein de véritables équipes de trois marins ou plus. De tels groupes d'individus étaient organisés autour d'un leader. L'équipage d'un navire de long cours étant un modèle de hiérarchie, c'est tout naturellement que l'on pouvait identifier deux niveaux dans les équipes parties de Marennes. Très homogènes, les groupes de trois marins de Seudre (14 \%) se constituaient autour de deux matelots dans la plupart des cas. Les ordonnances faisant obligation aux capitaines, sous peine de perdre leur exemption de service, d'embarquer $20 \%$ de mousses et $10 \%$ de novices, on imagine sans mal que ceux-ci encourageaient les matelots de confiance à leur ramener un ou deux jeunes. Un véritable parrainage s'organisait. Le capitaine respectait la réglementation et s'assurait des relais de pouvoir à bord. Sur le Sartine armé pour l'Inde à Bordeaux, en septembre 1776, le capitaine Touronnet enrôlait ainsi les matelots Pierre Dugué (Mornac, 1752; 1768 à 1780) ${ }^{26}$ et Auguste Boisson (Mornac, 1752; 1765 à 1777) ${ }^{27}$ qui avaient fait le voyage depuis la Seudre avec Charles Lajoye (L'Éguille, 1755; 1771 à 1777) ${ }^{28}$ embarqué comme novice. Seul Dugué devait revenir, rappelant que s'embarquer en compagnie était aussi une forme d'assurance, offrant à la famille du disparu un témoin précieux à son travail de deuil. Avec les équipes formées de quatre marins et plus, (38 \%), le matelot n'était plus automatiquement

22. SHM Rochefort, Registre des mousses de Marennes (1760-1766), 10P3.1 $\mathrm{f}^{\circ} 63 \mathrm{n}^{\circ} 85 \mathrm{sqq}$.

23. SHM Rochefort, Registre des novices de Marennes (1764-1787), 10P3.4 $\mathrm{f}^{\circ} 63 \mathrm{n}^{\circ} 31 \mathrm{sqq}$.

24. SHM Rochefort, Registre des mousses de Marennes (1760-1766), 10P3.1 f 269 $\mathrm{n}^{\circ} 127 \mathrm{sqq}$.

25. SHM Rochefort, Registre des novices de Marennes (1764-1787), 10P3.4 $\mathrm{f}^{\circ} 303 \mathrm{n}^{\circ} 70$.

26. SHM Rochefort, Registre des novices de Marennes (1764-1787), 10P3.4 $\mathrm{f}^{\circ} 612 \mathrm{n}^{\circ} 7 \mathrm{sqq}$.

27. SHM Rochefort, Registre des mousses de Marennes (1760-1766), 10P3.1 f 474 $\mathrm{n}^{\circ} 29 \mathrm{sqq}$.

28. SHM Rochefort, Registre des mousses de Marennes (1766-1787), 10P3.3 f 158 $\mathrm{n}^{\circ} 21 s q q$. 
l'initiateur du projet. Logiquement, la taille de l'équipe croissait avec la qualification du leader. Hiérarchie et dépendance prenaient le relais de la solidarité qui animait les petits groupes. Les huit marins du quartier enrôlés sur le long-courrier La Reine Ester, armé pour Saint Domingue en 1775 à La Rochelle, représentaient le tiers d'un équipage composé de 22 hommes, sous les ordres du capitaine Élie Chevroux, un Bourcefrançais " habitué " à La Rochelle. Les 34 navires où l'on comptait plus de trois marins de la Seudre offraient ainsi un dégradé de situations, illustrant le vieux réflexe de naviguer entre soi qui s'exprimait encore, au coup par coup, sur les derniers morutiers armés de Chaillevette à la Tremblade ${ }^{29}$.

De telles expéditions étaient parfois initiées en Seudre. Ainsi en 1765, les rôles d'armement du quartier de Marennes signalaient le départ du longcourrier Le Père de Famille (300 tonneaux) destiné à La Rochelle où il devait être " réarmé pour les Isles ". À son bord, 35 marins : 31 du Quartier et 4 Royannais ${ }^{30}$. Cet exemple renvoyait aux liens familiaux unissant les armateurs et les capitaines. L'histoire de Jacques Conte, originaire de Chaillevette et devenu armateur bordelais juste avant la Révolution est là pour le rappeler ${ }^{31}$. Le classement des armateurs de La Rochelle, effectué par Patrick Villiers suivant les armements 1775-1777 32 , en apporte d'autres preuves. Dans le groupe des propriétaires de plus de trois armements, on retrouvait Suidre (capitaines Marennais) et Garesché (négociants à Nieulle sur Seudre) avec chacun 11 navires. Le quatrième groupe, armateurs d'un navire unique, comprenait Ranson (étude notariale de Marennes) et Bourgine (capitaines de La Tremblade). Les routes de la ville, empruntées par les marins, épousaient les ramifications de réseaux économiques et d'arbres généalogiques qui faisaient descendre cette sève humaine, aux racines d'une bourgeoisie fière de ses origines et de sa réussite.

Ces pratiques rappelaient la constitution des équipages morutiers mais elles n'étaient ni aussi régulières ni aussi fréquentes. Contrairement aux " terre neuvas ", capitaines et armateurs recrutant en Seudre ne savaient pas avec précision quand et sur qui ils pourraient compter. Dans ce monde de l'oralité, les engagements individuels pouvaient déboucher sur des trajets complexes à démêler. Reflet des rôles d'armement (ou de désarmement) des ports extérieurs au quartier, les matricules de Marennes révè-

29. À ce sujet, voir notre article : « Les derniers morutiers d'Aunis et Saintonge (1762-

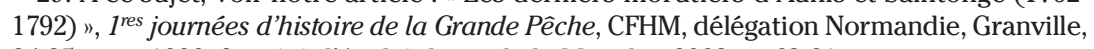
24-25 sept. 1999, Société d'Archéologie de la Manche, 2003, p. 63-81.

30. SHM Rochefort, Rôles d'armement du quartier de Marennes, 10P5.17 n²0, année 1765.

31. Sur l'ascension de la famille Conte, on lira avec profit É. Saugera (op. cit.), qui présente une généalogie de cette famille huguenote p. 130 sqq. Il évoque la carrière d'armateur de Jacques entre les années 1780 et 1803, où il perd ses quatre derniers navires et réinvestit dans la vigne le reste de ses capitaux.

32. VILLIERS, Patrick, "Peut-on parler d'une flotte littorale? L'exemple des flottes coloniales de Bordeaux, La Rochelle et Nantes de 1778 à 1789 ", dans Les sociétés littorales du Centre Ouest atlantique, GERHICO, Rochefort colloque de 1995, Poitiers, SAO, 1996, p. 309. 
lent certaines discordances au niveau des dates d'embarquement. Les mêmes écarts affectent les fins de campagnes (débarquement/désarmement). Certes, la concordance, jour pour jour, domine. Ainsi, $25 \%$ des embarquements enregistrés à Marennes étaient datés du même jour que l'armement du navire, tandis que $65 \%$ avaient lieu dans une fourchette de plus ou moins 15 jours. Moins nette, la concordance s'impose aussi du côté des débarquements puisque 15 \% étaient enregistrés à la date du désarmement et $45 \%$ prenaient place dans la fourchette des 15 jours. Il reste que la fréquence des écarts supérieurs à 15 jours est non négligeable, certains étant supérieurs à 150 jours, soit 5 mois... Ces enregistrements, loin d'être aberrants, offrent de nos trajets professionnels quelques éclairages intéressants. Ainsi, pour un même voyage, l'attention est immédiatement attirée par les différences entre ports d'armement (rôle) et d'embarquement (matricule). Dans 50 \% des cas, les deux lieux diffèrent, alors que c'est tout à fait marginal (8 \%) pour les embarquements dont la date coïncide avec l'armement du navire.

Le Trembladais Jean Allard (La Tremblade, 1750 ; 1780 à 1797) est ainsi enregistré : « le 24 avril 1786, permis pour Lorient, le 15 mai 1786 à Angolle, lieutenant à 45 lt. Sur Le Bonhomme Richard, capitaine Pelletier, débarqué

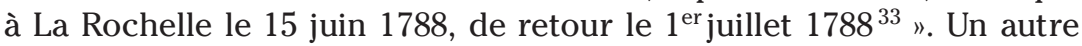
Trembladais, André Papin ${ }^{34}$ (La Tremblade, 1747; 1767 à 1803), est second capitaine sur ce navire. Le rôle d'armement correspondant à cette campagne de traite ${ }^{35}$ est daté de La Rochelle, le 16 octobre 1786. Cinq mois se sont écoulés entre l'embarquement à Lorient, enregistré à Marennes (15 mai), et l'armement, dont le rôle a été rédigé à La Rochelle (15 octobre). En fait, ces deux officiers du quartier ont gagné la Bretagne Sud pour prendre leur poste sur un navire, armé quelques semaines plus tard en Aunis. En atteste l'équipage enrôlé à La Rochelle, qui compte 39 Bretons sur 71 marins. Par ailleurs, deux matelots, un novice et un mousse du quartier de Marennes, ont bien été enrôlés sur ce navire de traite. Dans leur cas, les matricules enregistrent un embarquement à La Rochelle, le 16 octobre. L'ensemble de ce dossier est exemplaire d'une opération complexe d'armement. C'est en 1786 que ce navire entrait dans le capital des armateurs du centre-ouest et c'est pourquoi des membres de l'état-major se sont rendus à Lorient pour en prendre " livraison " et peut-être en vérifier l'état, en mai. Conduit à La Rochelle par un équipage réduit, sans doute composé des seuls Bretons, il est définitivement armé en traite en octobre 1786, grâce à l'enrôlement de Rochelais et de Saintongeais. À l'issue du voyage, il faut encore noter que, si le désarmement intervient le 25 mai 1788 à La Rochelle, le bureau de Marennes date le débarquement des quatre hommes d'équipage du 15 mai, soit un mois avant celui des deux officiers, le 16 juin.

33. SHM Rochefort, Matricules des novices de Marennes 10P3.4 f $314 \mathrm{n}^{\circ} 105$.

34. SHM Rochefort, Matricules des matelots et officiers mariniers de Marennes 10P3.9 $f^{\circ} 96 \mathrm{n}^{\circ} 135$

35. SHM Rochefort, Rôles d'armement de bureau de La Rochelle, année 1786, 6P39, $n^{\circ} 40$. 
Pressés d'être libérés, les marins étaient congédiés avant le désarmement, à la différence des officiers, qu'un rôle autant commercial que hiérarchique à bord mettait dans l'obligation de rendre des comptes.

Les sources maritimes permettent donc de cerner les trajets professionnels des marins ruraux. Pour 80 \% d'entre eux, le trajet vers Bordeaux ou La Rochelle était un acte collectif même si chacun n'intégrait pas au même moment de l'armement du navire. La parole d'un capitaine ou d'un maître d'équipage était le moteur principal du déplacement. Partir pour la ville était moins une aventure que le respect d'un engagement, le signe d'une intégration réussie, réalisée par les aînés au profit des plus jeunes. Pourtant, 20 \% des inscrits partaient seuls, en quête d'une embauche, souvent à bord des mauvais bateaux avec les mauvais capitaines, vers les destinations risquées de la traite ou de l'Inde. C'était le lot des laissés-pourcompte de la société littorale. Le fait de s'être déplacé pour rien, rapporté par le laconique "n'a pas embarqué ", pouvait dès lors être considéré comme un moindre mal.

Dans une France d'Ancien Régime marquée par la faible mobilité géographique, le mode de vie des marins ruraux engagés dans l'économie atlantique détonne. Pour 4 à $5 \%$ des habitants du quartier de Marennes, les déplacements étaient monnaie courante. À l'Aunis toute proche, Bordeaux disputait la main d'œuvre de la Seudre, la frontière des bassins d'emplois concurrents épousant le cours du fleuve. Les archives de la marine donnent le point de vue strictement professionnel de trajets fortement organisés. Elles révèlent un ensemble de migrations temporaires, pensées collectivement à défaut d'être toujours réalisées simultanément, par l'équipe enrôlée à bord d'un même navire. Pour chacun, l'essentiel était d'être exact au rendez-vous de la préparation d'une expédition, quelle que soit l'étape à laquelle on rejoignait le bord. Les trajets vers la ville étaient la traduction matérielle d'engagements pris d'un voyage à l'autre. À titre personnel, chacun s'assurait d'enchaîner plusieurs contrats auprès d'une même entreprise, au sein d'une même équipe. Le système préservait les chances de promotion, des apprentis aux matelots et des matelots vers la maistrance. À titre collectif, le système avait un double mérite. Il assurait la reproduction professionnelle, en entrainant à la suite des matelots, les jeunes du littoral, issus de familles maritimes ou non. En retour, les déplacements collectifs ménageaient les solidarités villageoises, triomphant de la défiance des familles de terriens. Moins d'un marin sur cinq partait seul, sans assurance de trouver un contrat. C'était le lot des laissés-pour-compte de la vie maritime, venus trop tard au métier, sans appui. Du village à la ville, les conditions du trajet reflétaient donc le degré d'intégration du marin, dans un monde professionnel aux règles strictes quoique non écrites. 


\section{RÉSUMÉ}

Grâce aux registres matricules des Classes, il est possible d'étudier les migrations temporaires induites par l'activité maritime des grands ports de l'Atlantique. Dans les deux dernières décennies du XVIII ${ }^{\mathrm{e}}$ siècle, les gens de mer ruraux de l'estuaire de la Seudre partagent ainsi leurs trajets entre les bassins d'emploi de Bordeaux et de La Rochelle, attirés par l'essor du trafic négrier déconsidéré par la main-d'œuvre locale qui préfère le commerce en droiture. Dans les familles de marins, la reproduction sociale est forte, alors que dans les milieux terriens, le large est surtout une destinée de cadets, de bras que les travaux des champs ne parviennent pas à absorber. Ces déplacements sont le plus souvent pensés collectivement. Ils s'accomplissent en équipes, constituées à partir de solidarités villageoises ou professionnelles : en général, un ou deux matelots expérimentés s'engagent au terme d'un voyage à ramener de leur terroir un novice pour la prochaine expédition. Ce système favorisait la mobilité sociale tout en ménageant la défiance des familles paysannes.

\section{ABSTRACT}

Thanks to the registres matricules des Classes, it is possible to study temporary migrations induced by maritime activity in the great harbours of the Atlantic. In the last two decades of the $18^{\text {th }}$ Century, the rural seamen of the estuary of the Seudre share their journeys between the busy areas of Bordeaux and La Rochelle, attracted by the slave trade which was not well considered by local working force, the latter preferring straightforward, lawful, commerce. Among the families of sailors, the social reproduction is high, whereas among the land-living people, the sea is essentially a destiny for the youngest of the families, for arms agricultural labour cannot absorb. These journeys are usually thought collectively. They're done in teams constituted from village or professional solidarities: in general, one or two experimented sailors engage themselves to bring back at the end of an expedition a novice from their community for their next expedition. This system favoured social mobility while sparing the defiance of peasant families. 